\title{
New bounds and estimates for porous media with rigid perfectly plastic matrix
}

\author{
Nicolas Bilger $^{\text {a,b }}{ }^{\text {François Auslender }}{ }^{\text {a }}$, Michel Bornert ${ }^{\text {a }}$, Renaud Masson ${ }^{b}$ \\ a Laboratoire de mécanique des solides, École polytechnique, CNRS, 91128 Palaiseau cedex, France \\ b Électricité de France, site des Renardières, route de Sens, Écuelles, 77818 Moret sur Loing, France
}

\begin{abstract}
We derive new rigorous bounds and self-consistent estimates for the effective yield surface of porous media with a rigid perfectly plastic matrix and a microstructure similar to Hashin's composite spheres assemblage. These results arise from a homogenisation technique that combines a pattern-based modelling for linear composite materials and a variational formulation for nonlinear media.
\end{abstract}

solids and structures / porous media / bounds / perfect plasticity

\section{Nouvelles bornes et estimations pour les milieux poreux à matrice rigide parfaitement plastique}

Résumé On présente de nouvelles bornes rigoureuses et estimations autocohérentes pour la surface de plasticité macroscopique de milieux poreux à matrice rigide parfaitement plastique dont la microstructure s'apparente à l'assemblage des sphères composites de Hashin. Elles sont déduites d'une technique d'homogénéisation qui combine une approche par motifs pour les composites linéaires et une formulation variationnelle pour les milieux non linéaires.

solides et structures / milieux poreux / bornes / plasticité parfaite

\section{Version française abrégée}

De nouvelles bornes et estimations autocohérentes pour la surface de plasticité de milieux poreux dont la microstructure peut être décrite par un assemblage de sphères composites de Hashin sont obtenues par l'association d'un principe variationnel pour l'homogénéisation des comportements non linéaires $[5,10]$ et d'une méthode d'homogénéisation linéaire reposant sur la notion de motif morphologique représentatif [11,12], qui permet une description améliorée des interactions dans l'environnement immédiat d'un pore.

E-mail addresses: bilger@1ms.polytechnique.fr (N. Bilger); auslende@1ms.polytechnique.fr (F. Auslender); bornert@1ms.polytechnique.fr (M. Bornert); Renaud.Masson@edf.fr (R. Masson). 
La démarche est la suivante. Les équations du problème local non linéaire sont traitées selon les principes de la méthode sécante modifiée [10], qui rejoint l'approche variationnelle de Ponte Castañeda [5]. Contrairement aux mises en œuvre usuelles de ces principes, on fait ici appel, comme dans [13], à un composite linéaire de comparaison multiphasé, avec plusieurs modules sécants dans la matrice, et non pas un seul module constant. Plus précisément, la microstructure est classiquement [2,3] supposée s'apparenter à l'assemblage des sphères composites de Hashin [4], formées d'un pore sphérique entouré d'une gaine de matrice, de taille variable mais de fraction volumique égale à la porosité globale et réparties aléatoirement dans un volume élémentaire représentatif de façon à couvrir tout l'espace (Fig. 1a). Dans le but de mieux représenter l'hétérogénéité radiale des champs mécaniques induite par la plasticité, les couronnes de matrice sont discrétisées en $N$ couches concentriques (Fig. 1b) et l'on affecte à chaque couche $r$ un module sécant différent $\mu_{r}$. Le milieu linéaire de comparaison ainsi obtenu admet une description par motifs morphologiques représentatifs [11], les motifs étant les sphères composites multicouches ainsi définies. L'homogénéisation de son comportement peut être abordée analytiquement au travers de l'approche variationnelle présentée dans [11,12], qui conduit, sous l'hypothèse réaliste que les motifs sont répartis de manière isotrope, au problème d'une d'inclusion composite multicouche noyée dans un milieu de référence homogène sous chargement homogène à l'infini (Fig. 1c), dont la solution analytique est connue [15]. Suivant le choix du milieux de référence, une borne supérieure [11,12] ou une estimation autocohérente $[13,14]$ des propriétés du composite linéaire, et par suite de la surface de plasticité du milieu poreux, sont obtenues. Les équations à traiter sont résumées par le système (3), où $\sigma_{0}$ est la contrainte d'écoulement de von Mises de la matrice, $T$ le taux de triaxialité macroscopique (rapport entre contrainte moyenne $\Sigma_{\mathrm{m}}$ et équivalente $\Sigma_{\text {eq }}$ ) et la fonction $F$ fait référence à l'homogénéisation du milieu linéaire de comparaison. Les dérivées partielles de cette dernière donnent accès au moment d'ordre deux $\left\langle\varepsilon_{\text {eq }}^{2}\right\rangle_{r}$ de la déformation dans les couches selon (2) et sont calculées numériquement.

La résolution de (3) par une méthode de point fixe pour plusieurs valeurs de $T$ permet de construire la fonction seuil point par point. Les résultats pour une porosité $f$ de $1 \%$ et $5 \%$ sont donnés en Fig. 2 . Avec un nombre de couches suffisamment grand $(N \geqslant 10)$ et pour un chargement purement hydrostatique, on converge vers la solution exacte (Fig. 2b), également donnée par le critère de Gurson. Avec une seule couche, on retrouve pour tout $T$ la borne de Hashin et Shtrikman non linéaire (NLHS+). Sous chargement purement déviatorique, la discrétisation n'a qu'un faible effet (Fig. 2a), mais elle permet d'améliorer les bornes et estimations existantes (Fig. 2c) dont en particulier celles à caractère analytique dues à Gărăjeu et Suquet $[3,8]$. Le gain est plus net pour des triaxialités intermédiaires. L'estimation autocohérente est très proche de la borne supérieure, mais s'en écarterait pour une porosité plus élevée.

\section{Introduction}

During the last 25 years, noticeable progress has been made in the modelling of nonlinear porous media. Gurson, in his pioneering work on a hollow rigid plastic sphere [1], exhibited a macroscopic yield function which involves, besides classical parameters such as the equivalent von Mises stress $\Sigma_{\text {eq }}$ and the yield stress $\sigma_{0}$, the porosity $f$ and the hydrostatic stress $\Sigma_{\mathrm{m}}$ :

$$
\left(\frac{\Sigma_{\text {eq }}}{\sigma_{0}}\right)^{2}+2 f \cosh \left(\frac{3 \Sigma_{\mathrm{m}}}{2 \sigma_{0}}\right)-1-f^{2}=0
$$

This criterion has the noticeable property to be an upper bound [2,3] for the particular class of microstructures described by Hashin's [4] composite spheres assemblage (CSA), made up of spherical pores surrounded by concentric shells of matrix, in different sizes but with the same volume fraction, and distributed in such a way that they fill the whole space (Fig. 1a). Moreover, for purely hydrostatic loads, it 


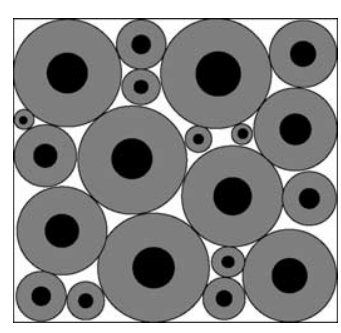

(a)

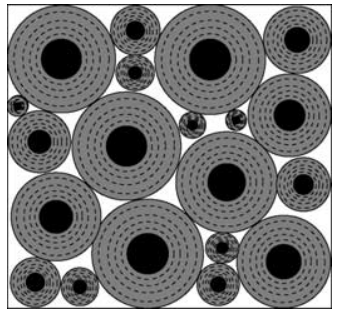

(b)

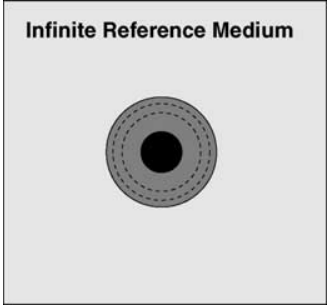

(c)

Figure 1. Composite spheres assemblage (a); discretisation into layers (b); multilayered inclusion (c).

Figure 1. Assemblage de sphères composites (a); discrétisation en couches (b); inclusion multicouche (c).

gives the exact solution. Another rigorous upper bound for the macroscopic plastic yield surface has been proposed later on by Ponte Castañeda [5] and Michel and Suquet [6]; it is based on an extension to nonlinear behaviour of the linear Hashin and Shtrikman upper bound and applies to any microstructure in which the spatial distribution of the pores is isotropic. It will be referred to by NLHS+. When the triaxiality factor $T=\Sigma_{\mathrm{m}} / \Sigma_{\text {eq }}$ goes to zero, this new bound improves on Gurson's criterion. However, for infinite triaxiality, Gurson's criterion keeps predominance. More recently, Gărăjeu [3] has derived another analytical upper bound that applies to Hashin's assemblage, using more refined admissible strain fields in the composite spheres than according to Gurson's analysis. His criterion is very similar to (1), except that the first term has to be multiplied by some complex coefficient $\tau(f)$. Since $\tau(f)<1$, his result, referred to as GSH+ in the following, improves on Gurson's criterion for non purely hydrostatic loads and gives the exact result for $T=\infty$. However, it lies slightly above NLHS + for $T=0$. Several authors $[7,8]$ have suggested to replace $\tau(f)$ by $1+\frac{2}{3} f$; the obtained estimate (GS in the following) of the yield function coincides then with NLHS+ for $T=0$ and with (1) for $T=\infty$, but there is no proof that such a relation yields an upper bound. In the present paper, new bounds and estimates for the yield function of porous media with a microstructure similar to Hashin's CSA are derived; they are consistent with the above mentioned best bounds for $T=0$ and $T=+\infty$ and improve on them for any other triaxiality.

We consider a representative volume element $\Omega$ of a such a porous medium. The homogeneous and isotropic matrix is rigid and perfectly plastic, follows the von Mises criterion, with yield stress $\sigma_{0}$, and obeys the normality rule. Under these assumptions and when $\Omega$ is submitted to macrohomogeneous boundary conditions, an effective behaviour of the porous material can be defined; it is rigid perfectly plastic and obeys the normality rule [9] as well. When, in addition, the spatial distribution of the pores is isotropic, the overall yield criterion depends only on the three invariants of the overall stress $\boldsymbol{\Sigma}$. The proposed models, alike those mentioned above, are neither sensitive to the third invariant nor to the sign of the pressure, so that the overall yield function depends only on $\Sigma_{\text {eq }}$ and $\left|\Sigma_{\mathrm{m}}\right|$. To construct it, we determine, for any imposed value of the triaxiality $T$, the overall stress that leads to some non vanishing overall strain $\boldsymbol{E}$, with arbitrary magnitude. The full yield function in the ( $\left.\left|\Sigma_{\mathrm{m}}\right|, \Sigma_{\text {eq }}\right)$ plane is then obtained when $T$ varies from 0 to $+\infty$.

We make use of the "modified secant procedure" proposed by Suquet [10] which is equivalent to Ponte Castañeda's variational procedure [5]. But unlike these authors, we are dealing with a "linear comparison composite" (LCC), in the sense of Ponte Castañeda, with a piecewise constant but non uniform distribution of shear moduli in the matrix. This enriched formulation of the comparison medium leads to a better description of the plastic flow in the nonlinear medium and is the key of the obtained improvements. The multiphase LCC is handled by an extension of the linear Hashin and Shtrikman procedure to microstructures described by means of morphologically representative patterns $[11,12]$, which leads to an (almost) analytical solution of the linear problem. The detail of the procedure is given in the next section and the results are commented and compared to above mentioned earlier models in the third one. 


\section{Nonlinear variational procedure based on multilayered composite spheres}

2.1. Modified secant approach. - If we assume for simplicity that the local deviatoric stress evolves in a proportional and monotonic way, the deformation theory of plasticity applies. The matrix being isotropic and incompressible, its local behaviour may be described by a secant constitutive law involving a local secant shear modulus $\mu\left(\varepsilon_{\text {eq }}\right)$ which depends at each point $\boldsymbol{x}$ of $\Omega$ on the local equivalent strain $\varepsilon_{\text {eq }}(\boldsymbol{x})$. The nonlinear problem can be simplified by replacing this distribution of local moduli by a piecewise uniform one, the secant moduli being equal to $\mu_{r}$ in the sub-domain $\Omega_{r}$ of the matrix. This defines the so-called LCC, which is an $N+1$ phase composite made up of the pores and the $N$ sub-phases $\Omega_{r}, r \in[1, N]$, in the matrix, with volume fractions $c_{r}$. According to the modified secant approach, the moduli $\mu_{r}$ should be related, through the local secant constitutive law of the matrix, to some reference strain equal to the secondorder moment of the local strain field in the LCC over the domain $\Omega_{r}: \varepsilon_{\text {eq }}^{r}=\sqrt{\left\langle\varepsilon_{\text {eq }}^{2}\right\rangle_{r}}$ where $\langle a\rangle_{r}$ is the volume average of $a$ over phase $r$. With such a choice, the effective properties of the LCC lead to an upper bound for the nonlinear composite [5], in terms of overall potentials. In the present case, one gets an outer bound for the yield surface. When the overall properties of the LCC are isotropic, with $\mu^{\text {hom }}$ and $k^{\text {hom }}$ the effective shear und bulk moduli, when an overall triaxiality $T$ is prescribed and when an overall equivalent strain $E_{\text {eq }}$ is imposed, the general relation for the second order moments given in [10] specialises into:

$$
\left\langle\varepsilon_{\text {eq }}^{2}\right\rangle_{r}=\frac{E_{\text {eq }}^{2}}{c_{r}}\left[\frac{\partial \mu^{\text {hom }}}{\partial \mu_{r}}+3\left(T \frac{\mu^{\text {hom }}}{k^{\text {hom }}}\right)^{2} \frac{\partial k^{\text {hom }}}{\partial \mu^{r}}\right]
$$

The nonlinear set of equations to determine the overall plastic limit is then given by (3), where $F$ provides the linear isotropic effective properties of the multiphase LCC. This system has to be solved in the $\mu_{r}$ by, for instance, an iterative procedure such as the classical fixed point method,

$$
\left\{\begin{array}{l}
\forall r \in[1, N], \quad \mu_{r}=\frac{\sigma_{0}}{3 \sqrt{\left\langle\varepsilon_{\text {eq }}^{2}\right\rangle_{r}}}, \quad\left\langle\varepsilon_{\text {eq }}^{2}\right\rangle_{r} \text { given by (2) } \\
\left\{k^{\text {hom }}, \mu^{\text {hom }}\right\}=F\left(c_{s}, \mu_{s}, s \in[1, N]\right) \\
\Sigma_{\text {eq }}=3 \mu^{\text {hom }} E_{\text {eq }} \quad \text { and } \quad \Sigma_{\mathrm{m}}=T \Sigma_{\text {eq }}
\end{array}\right.
$$

2.2. Multilayered composite spheres assemblage. - The full implementation of this algorithm requires the computation of the function $F$, which depends on the phase distribution. Alike other approaches mentioned above, we assume that the microstructure of the porous medium belongs to the class of composite spheres assemblages (Fig. 1a), made up of hollow spheres with a pore volume fraction equal to the macroscopic porosity, but different radii so that the whole domain $\Omega$ can be filled with such spheres. When this microstructure is submitted to plastic deformation, strong deformation gradients may appear near the pores. Under hydrostatic pressure, these gradients are mostly along the radii of the composite spheres. This observation suggests that, in order to describe more accurately the local plastic flow around the pores, it makes sense to discretise the matrix into concentric shells, as suggested in (Fig. 1b). As in [13], the multiphase LCC the present approach relies on is made of multilayered composite spheres, consisting of a pore surrounded by $N$ concentric layers. For simplicity, we assume that the geometry of the composite spheres of different sizes are homothetic. Thus the $r$ th phase, $r \in[1, N]$, of our multiphase LCC, with uniform shear modulus $\mu_{r}$, will be the union of all $r$ th layers of the composite spheres. Such a phase distribution can be described by means of "morphologically representative patterns" [11], a pattern being, in the present case, a multilayered sphere of a given size. The derivations presented in $[11,12]$, based on the Hashin and Shtrikman variational formulation, allow us to construct bounds and generalised self-consistent estimates $[13,14]$ for the effective properties of such composites. It is shown that, under the assumption of an isotropic distribution of the centres of the patterns, the effective properties can be deduced from the 
local fields that arise in such a multilayered inclusion embedded in an infinite linear isotropic reference medium (Fig. 1c), when it is submitted to homogeneous strain or stress conditions at infinity. Estimates of the effective shear (resp. bulk) modulus are obtained from the ratio between the average deviatoric (resp. hydrostatic) stress and the average deviatoric strain (resp. dilatation) in the composite inclusion. When the infinite medium has the properties of the stiffest layer, these estimates are indeed upper bounds for $\mu^{\text {hom }}$ (resp. $k^{\text {hom }}$ ), whereas when it has the estimated effective properties, so-called generalised self-consistent estimates are generated.

The multilayered composite inclusion problem can be solved analytically. The relations given in [15] provide exact and easy to implement expressions for the local and average stresses and strains in the layers, which can be extended to incompressible phases or to voids, as required for the present application. The combination of this solution and the system (3) finally leads to an outer bound and a self-consistent estimate for the effective yield surface of the nonlinear porous material. Note first that the derivatives in (2) are computed numerically because the analytical relations available from [15] are too complex. Second, the initial assumption of proportional loading does not restrict the generality of the results, since the obtained local and overall stresses do not depend on the magnitude of the arbitrarily small overall strain. This property could also be justified more rigorously within a formalism using dissipation potentials [9], not to be addressed here.

\section{Results}

The new bound $(\mathrm{N} \phi+)$ obtained for $1 \%$ porosity with $\mathrm{N}=1,2,5$ and 20 layers of same thickness as well as the new self-consistent estimate obtained with 20 layers $(20 \phi \mathrm{sc})$ are compared on Fig. $2(\mathrm{a}$ and b) to the Gurson model and the Hashin and Shtrikman upper bound NLHS+. The fact that $1 \phi+$ and NLHS+ coincide clearly results from the fact that the linear upper bound involved by both approaches are identical. With more layers, the new bound always improves on NLHS+. For a purely hydrostatic load, $(\mathrm{N} \phi+)$ converges to the Gurson model when $\mathrm{N}$ is increased. With 10 layers the discrepancy is for instance lower than $1 \%$. This result can easily be predicted from theoretical arguments since the local fields in the CSA are known exactly for a purely hydrostatic load: within any composite sphere, the displacement fluctuation is radial so that the stress and strain components in a local spherical coordinate system depend only on the distance $r$ to the centre of the corresponding pore. For an incompressible matrix, the displacement fluctuation in the matrix varies like $1 / r^{2}$, the local equivalent strain like $1 / r^{3}$ and the local secant modulus like $\sigma_{0} r^{3}$. Such a distribution can be approached exactly by the LCC when the discretisation is refined, so that the fields in the nonlinear composite can be described exactly within the secant approach. One can check that

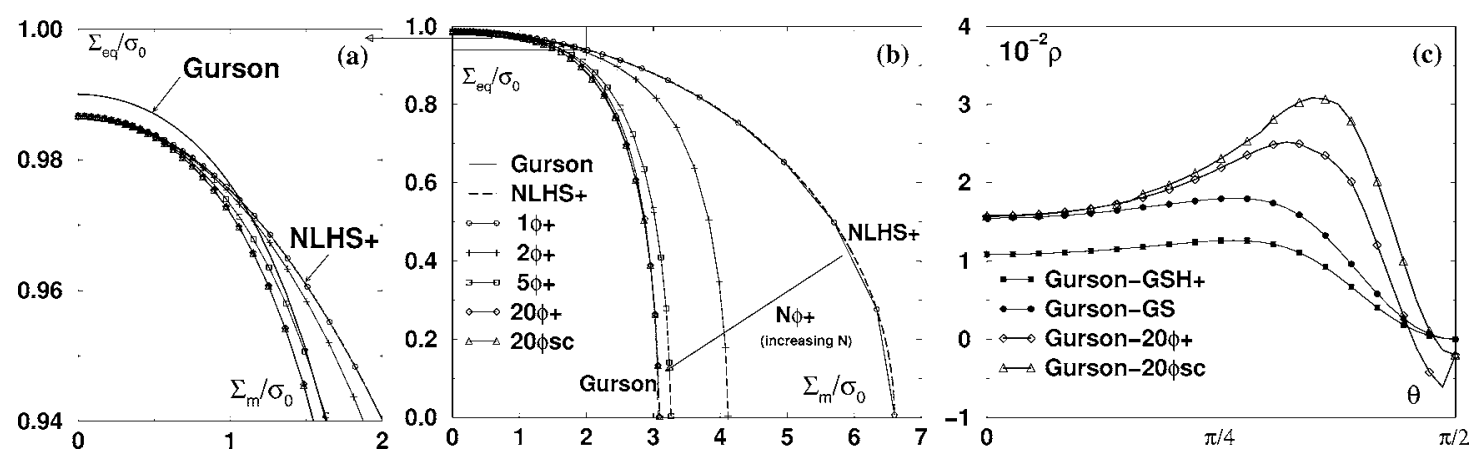

Figure 2. (b) Yield surfaces for $f=1 \%$. (a) zoom for small $T$. (c) Deviation of yield surfaces with respect to Gurson's criterion for $f=5 \%$ in $(\rho, \theta)$ polar coordinates of the $\left(\Sigma_{\mathrm{m}}, \Sigma_{\text {eq }}\right)$ plane.

Figure 2. (b) Surfaces seuils pour $f=1 \%$. (a) zoom pour T petit. (c) Écarts entre surfaces seuils et critère de Gurson pour $f=5 \%$ en coordonnées polaires $(\rho, \theta)$ du plan $\left(\Sigma_{\mathrm{m}}, \Sigma_{\mathrm{eq}}\right)$. 
the numerical solution of (3) does indeed converge to this distribution of secant moduli. For intermediate triaxialities, the new bound derived with 20 layers is always somewhat lower than Gurson's criterion. For a purely deviatoric load, the latter is definitively too stiff; the new bound is very close to NLHS+ and seems to be independent of the discretisation. In fact, it is not: one can plot in this case the evolution of $\mu_{s}$ with respect to $r$ and observe a non constant evolution which becomes more and more significant when the porosity is increased. Thus the new bound is always softer than NLHS+ even if the difference can be neglected in practice for small porosity. As expected, the self-consistent estimate is always within the bound for a similar discretisation, but very close to it for small porosity.

The plot on Fig. 2c provides, for a porosity of 5\%, a comparison between the new bound $(20 \phi+)$ and estimate $(20 \phi \mathrm{Sc})$ calculated for 20 layers, and the best up to now available rigorous bound $(\mathrm{GSH}+)$ and estimate (GS) mentioned in the introduction. In order to emphasise the improvements, it gives, as a function of the polar angle $\theta=\arctan (T)$ in the $\left(\left|\Sigma_{\mathrm{m}}\right|, \Sigma_{\text {eq }}\right)$ plane, the difference between the 'radii' $\rho=\sqrt{\Sigma_{\mathrm{m}}^{2}+\Sigma_{\text {eq }}^{2}}$ predicted by these models and that given by Gurson's criterion. Except in a small zone at a very large triaxiality where the number of layers is too small, we observe that $20 \phi+$ improves on $\mathrm{GSH}+$ and $20 \phi \mathrm{sc}$ improves on GS. Moreover, it is worth noting that the $20 \phi+$ bound is tighter than the GS estimate. When the triaxiality tends to infinity, we observe that the new bound and estimate tend to the Gurson bound but are less accurate than the other models. This discrepancy can be reduced with an increased discretisation, but at a significant numerical cost. The $\mathrm{GSH}+$ remains the best fully analytical bound valid for any CSA. When the composite spheres are isotropically distributed, $\mathrm{N} \phi+$ is more restrictive. However, in this case, the less accurate but fully analytical and very simple GS criterion seems to be a reasonable model for more practical applications.

Acknowledgements. The authors are grateful to P. Suquet and A. Zaoui for their comments and advices.

\section{References}

[1] A.L. Gurson, Continuum theory of ductile rupture by void nucleation and growth. Part 1 - Yeld Criteria and flow rules for porous ductile media, J. Eng. Mat. Tech. 99 (1977) 2-15.

[2] G. Perrin, Contribution à l'étude théorique et numérique de la rupture ductile des métaux, Thèse École polytechnique, 1992.

[3] M. Gărăjeu, Contribution à l'étude du comportement non linéaire de milieux poreux avec ou sans renforts, Thèse Université Aix-Marseille 2, 1995.

[4] Z. Hashin, The elastic moduli of heterogeneous materials, J. Appl. Mech. 29 (1962) 143-150.

[5] P. Ponte Castañeda, New variational principles in plasticity and their application to composite materials, J. Mech. Phys. Solids 40 (1992) 1757-1788.

[6] J.C. Michel, P. Suquet, The constitutive law of nonlinear viscous and porous materials, J. Mech. Phys. Solids 40 (1992) 783-812.

[7] J.B. Leblond, G. Perrin, P. Suquet, Exact results and approximate models for porous viscoplastic solids, Int. J. Plast. 10 (1994) 213-235.

[8] M. Gărăjeu, P. Suquet, Effective properties of porous ideally plastic or viscoplastic materials containing rigid particles, J. Mech. Phys. Solids (1996) 1-30.

[9] P. Ponte Castañeda, P. Suquet, Nonlinear composites, Adv. Appl. Mech. 34 (1998) 171-302.

[10] P. Suquet, Overall properties of nonlinear composites: a modified secant moduli theory and its link with Ponte Castañeda's nonlinear variational procedure, C. R. Acad. Sci. Paris, Série II 320 (1995) 563-571.

[11] C. Stolz, A. Zaoui, Analyse morphologique et approches variationnelles du comportement d'un milieu élastique hétérogène, C. R. Acad. Sci. Paris, Série II 312 (1991) 143-150.

[12] M. Bornert, C. Stolz, A. Zaoui, Morphologically representative pattern-based bounding in elasticity, J. Mech. Phys. Solids 44 (1996) 307-331.

[13] M. Bornert, E. Hervé, C. Stolz, A. Zaoui, Self-consistent approaches and strain heterogeneities in two-phase elastoplastic materials, Appl. Mech. Rev. 47 (1994) 66-76.

[14] M. Bornert, Morphologically representative pattern-based modelling in elasticity, Proc. Cont. Mod. Discr. Syst. 8 (1996) 123-131.

[15] E. Hervé, A. Zaoui, $N$-layered inclusion-based micromechanical modelling, Int. J. Engrg. Sci. 31 (1993) 1-10. 